\title{
Optical bottle microresonators: cleaning-up the spectral mess
}

\author{
M. N. Zervas \\ Optoelectronics Research Centre, University of Southampton, Southampton SO17 1BJ, U.K. \\ (mnz@orc.soton.ac.uk)
}

\begin{abstract}
We have demonstrated robust and accurate methods of selecting whispering gallery modes in optical bottle microresonators (BMR) by accurately placing high-index liquid drops and inscribing micro-scars on BMR's surface by focused ion beam milling. BMRs with cleaned-up transmission spectra and superior performance are obtained.
\end{abstract}

\section{Introduction}

Optical microresonator based devices have recently attracted considerable attention, promising an increasingly wide range of applications [1]. Among microresonators, microdisks [2], microspheres [3] and microtoroids [4] rely on the efficient excitation of whispering gallery modes (WGM), which are confined predominantly in one azimuthal plane and show well resolved spectral characteristics. A recently proposed truly 3-D optical microresonator, namely the bottle microresonator (BMR) [5]-[10], proves to be very promising for a number of applications such as cavity QED studies [9], strain [11] and optofluidic [12] sensors as well as add-drop multiplexers [13]. BMRs, however, exhibit particularly dense spectral features, which require considerable effort to be identified [10]. The rich spectral features are a result of the strongly broken degeneracy between WGMs with common azimuthal and differing axial mode numbers, as a result of the highly prolate shape. While dense spectral features are highly advantageous when bottle microresonators are used in cavity QED studies [9], they can potentially be a serious hindrance if BMRs were to be used in other applications such as refractometric optofluidic sensors. In this case, a cleaner spectrum with more easily identifiable and traceable spectral features would be desirable. Exploiting the distinctly different spatial intensity distributions of the non-degenerate resonances supported by such highly non-spherical microresonators, different approaches, such as using high-index prisms [14] and micro-droplets [10], have been used to differentially attenuate modes and clean-up the spectrum. However, these techniques are cumbersome, not accurately controlled and difficult to integrate in practical systems.

In this presentation, we describe different mechanisms, namely, a high-index liquid drop [10] and an accurately placed and controlled micro-scar [15][16] on the surface of the BMR - using high-precision focused ion beam (FIB) milling - to clean up the original dense spectrum. The former diffracts while the latter scatters light out of the resonator and preferentially attenuate a subset of the bottle modes. In the case of micro-scars, the shape, size and orientation of the scars can be controlled for optimum spectral clean-up. In addition, more than one micro-scar can be optimally placed in order allow only one bottle mode to survive.

\section{Device Fabrication and Characterization}

The "soften-and-compress" solid BMR fabrication technique [6][8] was employed to produce robust resonators. In this work, a standard telecom fiber (Corning SMF28) was used to fabricate BMRs with typical neck-to-neck distance $L b=350$ $\mu \mathrm{m}$, bottle diameter $D_{b}=170 \mu \mathrm{m}$ and stem diameter $D_{s}=125 \mu \mathrm{m}$. We have also extended this technique to produce highly-controllable, high performance hollow BMRs (HBMRs). The HBMRs were fabricated from a slightly pressurised glass capillary using a standard fusion splicer. This method has the advantage of being easy to implement, and yet very flexible. One end of the capillary was sealed, while the other was connected to a custom-built pressurisation system. In contrast with the technique reported in [17], which relies on high internal pressure only to form a microbubble, our technique uses moderate pressure, sufficient to counteract the surface tension in the softened glass, and relies entirely on the "compression" action of the splicer to form a highly-controllable-curvature "bottle" shape. HBMR s with typical dimensions of $D_{c}=218 \mu \mathrm{m}, D_{\mathrm{b}}=265 \mu \mathrm{m}$ and bottle length $L_{b}=550 \mu \mathrm{m}$ were fabricated. The BMRs were optically excited 
with a $2 \mu \mathrm{m}$ diameter tapered fiber. The micro-tapered fiber was in physical contact with the microresonator which, while lowering the loaded Q's, results in extremely stable operation. One of the pigtails of the excitation fiber taper was connected to a tuneable laser and the other pigtail to an InGaAs detector to measure the transmitted light. $Q$ factors of $\sim 10^{7}$ were obtained.

\section{BMR Spectral Clean-Up}

Figure 1(a) shows a high-index liquid drop [10] placed on the surface of the BMR. Figure 1(b), on the other hand, shows accurately placed and controlled micro-scars [15][16] on the surface of the BMR - using high-precision focused ion beam (FIB) milling. Both techniques are used to clean up the original dense BMR transmission spectrum.

Scars of different sizes and orientations were carved using the FIB system "Helios 600" (FEI Inc., Hillsboro, USA). FIB beam sizes smaller than $50 \mathrm{~nm}$ can be easily obtained, thus scar sizes can be controlled with excellent precision. Fig. 1 shows the SEM images of the gold-coated FIB-milled BMRs. In sample \#1 (Fig. 1(b2)) two scars were milled perpendicularly to the resonator axis and placed asymmetrically with respect to the BMR center. The first scar, with 5 $\mu \mathrm{m}$ width, $109 \mu \mathrm{m}$ length and $6 \mu \mathrm{m}$ depth, was placed $50 \mu \mathrm{m}$ away from the center. The second scar, with $5.5 \mu \mathrm{m}$ width, $27.5 \mu \mathrm{m}$ length and $6 \mu \mathrm{m}$ depth, was placed $83 \mu \mathrm{m}$ away from the center. In sample \#2 (Fig. 1(b1)), a single scar, with $5 \mu \mathrm{m}$ width, $50 \mu \mathrm{m}$ length and $6 \mu \mathrm{m}$ depth was carved parallel to the BMR axis and placed $50 \mu \mathrm{m}$ away from the center. The gold coating was removed after FIB milling was completed.

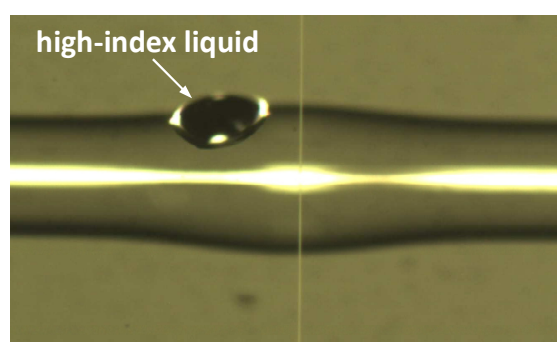

(a)

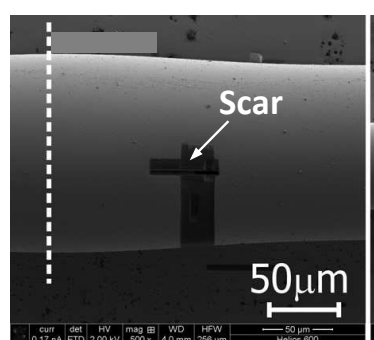

(b1)

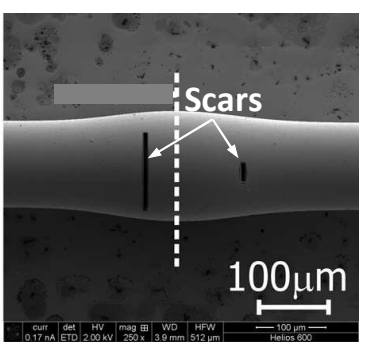

(b2)

Figure 1: BMR spectrum clean-up mechanisms: (a) high-index liquid drop and (b) FIB-milled scars on BMRs: (b1: sample \#1 with one scar parallel to the axis, b2: sample \#2 with two scars perpendicular to the axis.

The dense spectral features shown in Fig. 2(a) - top - are highly advantageous when bottle microresonators are used in cavity QED studies [9]. However, such spectral characteristics may be a serious hindrance if HBMRs were to be used as refractometric optofluidic sensors. In this case, a "cleaner" spectrum with more easily identifiable and traceable spectral features would be desirable. As it has already been discussed before [8], the rich spectral features are a result of the strongly broken degeneracy between whispering-gallery modes with common azimuthal and differing axial mode numbers, as a result of the highly prolate shape. In addition to different eigen-frequencies, these non-degenerate resonances exhibit substantially different spatial intensity distributions along the bottle length [8][9][10]. This property of the resonant modes can be exploited to differentially attenuate some of the modes according to axial mode-number and thereby substantially "clean up" the spectrum. This is similar to the modal filtering method applied to cm-sized crystalline disks using an auxiliary out-coupling prism [14]. Figure 2(a)-left shows the normal spectrum of the HMBR excited at the center. Figure 2(b)-left, on the other hand, shows the "cleaned-up" spectrum, with a loss element placed away from the center near the bottle edge. In this case the loss was introduced by depositing a small drop of high index liquid in different positions along the bottle length. The cleaner spectrum consists of modes that occupy the central part of the bottle without suffering the extra loss and $Q$ deterioration due to the loss element. Figure 2(c)-left shows detail of the cleaned-up and improved spectrum. HBMRs with "cleaned-up" spectral responses would provide tunable filters, optofluidic and other refractometric sensors with significantly improved performance.

Before FIB milling, rich and dense spectral characteristics are shown in Fig. 2(a)-right with the tapered fiber in the center of the BMR. In this case, all BMR modes can be potentially excited with amplitude that depends on their coupling strength with the micro-taper mode. Similarly dense spectra were obtained with the tapered fiber in different positions along the BMR length [8][10]. Fig. 2(b)-right and (c)-right show the corresponding spectra when WGMs in 
sample \#1 are excited with the microtaper placed at the center and $10 \mu \mathrm{m}$ off-center, respectively. It is shown that the spectra of the scarred BMR are substantially cleaner. As in the case of localized diffractive losses [10], this is due to the fact that only BMR modes concentrated around the resonator center survive with minimum losses, while modes which overlap or extend beyond the FIB scars experience severe scattering losses and are not excited efficiently. When the tapered fiber was placed beyond the region with two scars no sharp transmission resonances were observed. However, for a tapered-fiber position $\sim 120 \mu \mathrm{m}$ from the center, shown in Fig. 2(f)-right, sharp and well resolved periodic transmission notches appeared again.
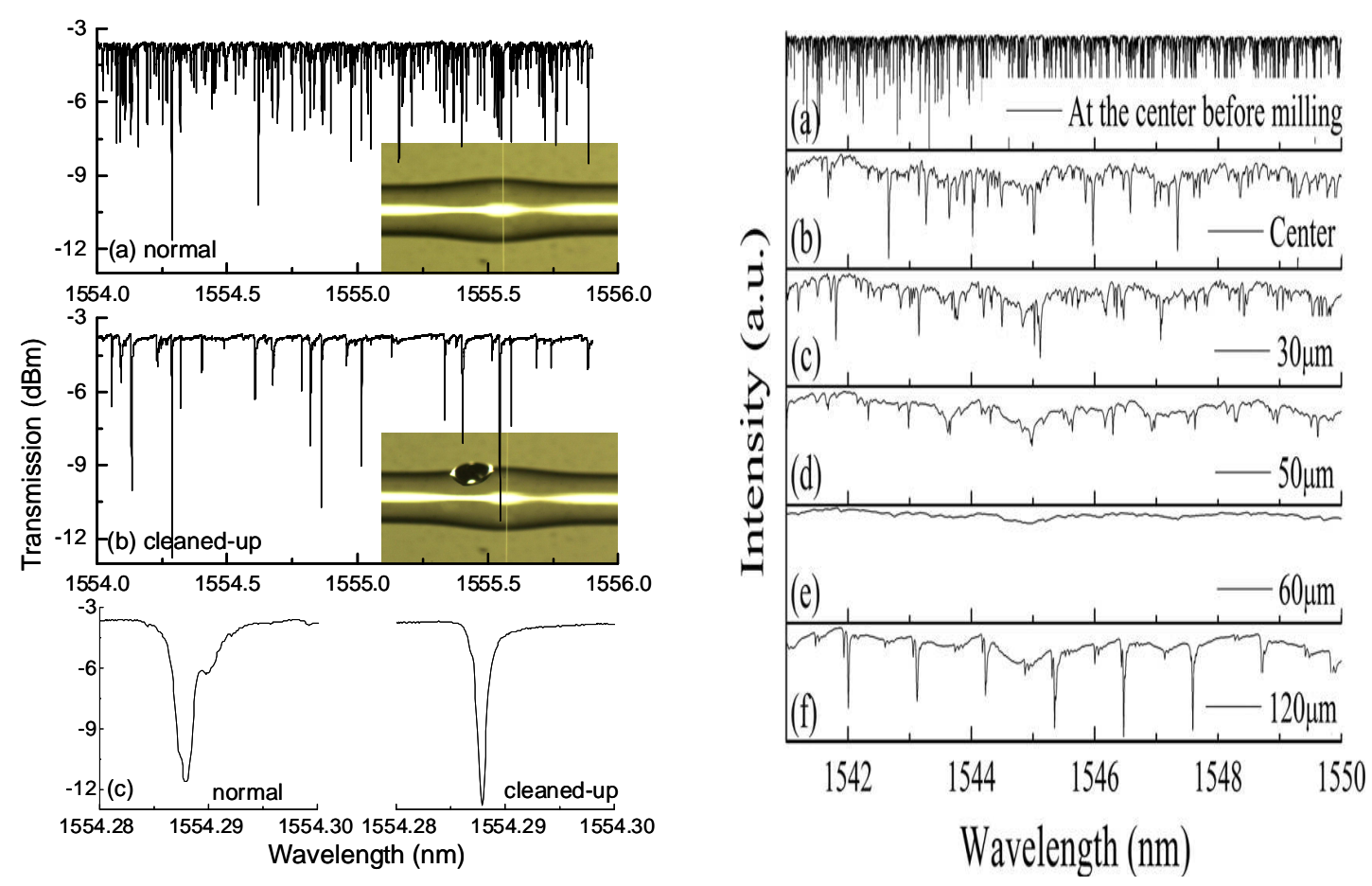

Figure 2: (a) Transmission spectra of the tapered fiber-coupled \#1 BMRs excited at the microresonator center (a) before microstructuring, after microstructuring at (b) the center; (c) $10 \mu \mathrm{m}$ off-center; (d) $120 \mu \mathrm{m}$ off-center; (e) Lorentzian fitting to a resonance group in Fig. 2 (d); (f) intensity distribution along the BMR axis when two modes are excited simultaneously.
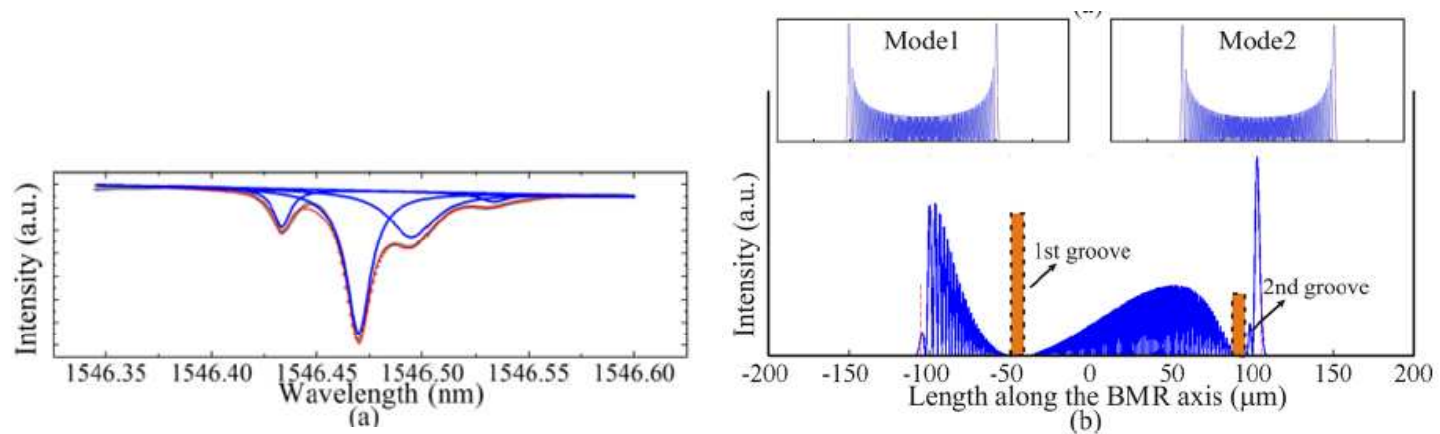

Figure 3: (a) Lorentzian line fitting of a resonance in Fig. 2(f) (right) and (b) intensity distribution along the BMR axis when two modes are excited simultaneously (the insets show the intensity distributions of the two individual beating modes). 
This is a unique feature of the two asymmetrically placed perpendicular scars of sample \#1 and was not observed in the case of the long longitudinal scar of sample $\# 2$ or the diffractive localized loss in Ref. [10]. Fig. 3(a) shows that each of the resonance groups of Fig. 2(f)-right constitutes several partially-overlapping Lorentzian resonances. Fig. 3(b) shows the intensity distribution along the BMR length when two bottle modes are excited simultaneously. The mode beating results in broad-enough power minima in two places along the length. When these minima coincide with the scars the effects of the scattering loss is minimized and the sharp resonances re-appear. In this way, well defined individual groups of modes, with large axial mode number, occupying a large section of the BMR can be efficiently excited. It should be noted that each mode alone would not have "tunneled" through the scars and survived, since their power minima (anti-nodes) occur over areas much narrower that the scar width ( 5um). It is demonstrated that BMR modes ca "work" together and survive the detrimental effects of localized scattering losses. Such modes are expected to play important role in sensing applications.

\section{Summary - Conclusions}

In conclusion, we have presented two efficient, controllable and robust methods of cleaning-up dense optical spectra in BMRs, using high-index drops and micro-scars produced by high precision FIB techniques. We have also demonstrated that a reduced group of modes can be predominantly selected with high-Q factor in BMRs with two asymmetrical scars. The shapes and positions of the milled scars can be chosen according to the application and the performance of microstructured BMRs can be improved by optimizing the scar shape size and orientation. This technique can be employed to clean up spectra in other types of non-spherical micro-resonators [4][14][17].

\section{References}

[1] K. J. Vahala, “Optical Microcavities”, Nature 424, 839 (2003).

[2] B. Gayral, J. M. Gerard, A. Lema1 tre, C. Dupuis, L. Manin, and J. L. Pelouard, "High-Q wet-etched GaAs microdisks containing InAs quantum boxes", Appl. Phys. Lett. 75, 1908 (1999).

[3] D. W. Vernooy, V. S. Ilchenko, H. Mabuchi, E. W. Streed, and H. J. Kimble, “High-Q measurements of fused-silica microspheres in the near infrared", Opt. Lett. 23, 247 (1998).

[4] D. K. Armani, T. J. Kippenberg, S. M. Spillane, and K. J. Vahala, "Ultra-high-Q toroid microcavity on a chip", Nature 421, 925 (2003).

[5] M. Sumetsky, “Whispering-gallery-bottle microcavities: the three-dimensional etalon," Opt. Lett. 29, pp. 8 (2004).

[6] M.N. Zervas, G. S. Murugan, and J. S. Wilkinson, "Demonstration of novel high-Q fibre WGM bottle microresonators", in Proceedings of the 10th Anniversary International Conference on Transparent Optical Networks (ICTON) (IEEE, Piscataway, NJ, 2008), Vol. 4, p. 58

[7] G. S. Murugan, J. S. Wilkinson, and M. N. Zervas, Conference on Lasers and Electro-Optics, 2009 and 2009 Conference on Quantum electronics and Laser Science Conference. CLEO/QELS 2009 (Optical Society of America, 2009), Paper No. JTuD87.

[8] G. S. Murugan, J. S. Wilkinson, and M. N. Zervas, "Selective excitation of whispering gallery modes in a novel bottle microresonator", Opt. Express 17, pp. 11916 (2009).

[9] M. Pollinger, D. O'Shea, F. Warken, and A. Rauschenbeutel, "Ultrahigh-Q Tunable Whispering-Gallery-Mode Microresonator", Phys. Rev. Lett. 103, 053901 (2009).

[10] G. S. Murugan, M. N. Petrovich, Y. Jung, J. S. Wilkinson, and M. N. Zervas, "Hollow-bottle optical microresonators", Opt. Express 19, pp. 20773 (2011).

[11] M. Sumetsky, Y. Dulashko, and R.S. Windeler "Super free spectral range tunable optical microbubble resonator", Opt. Lett. 35, 1866 (2010).

[12] Y. Sun, S.I. Shopova, C-S Wu, S. Arnold, and X. Fan, “Bioinspired optofluidic FRET lasers via DNA scaffolds”, PNAS 107, 16039 (2010).

[13] G.S. Murugan, J.S. Wilkinson, M.N. Zervas, "Optical excitation and probing of whispering gallery modes in bottle microresonators: potential for all-fiber add-drop filters" Opt. Lett. 35, 1893 (2010).

[14] A. Savchenkov, A. B. Matsko, D. Strekalov, V. S. Ilchenko, and L. Maleki, "Mode filtering in optical whispering gallery resonators", Electron. Lett. 41, pp. 495 (2005).

[15] M. Ding, G.S. Murugan, G. Brambilla, J.S. Wilkinson and M.N. Zervas "Robust Mode-Selection in Optical Bottle Microresonators" CLEO, paper CM2M.5 (2012).

[16] M. Ding G.S. Murugan, G. Brambilla, and M.N. Zervas, "Whispering gallery mode selection in optical bottle microresonators", Appl. Phys. Lett. 100, 081108 (2012).

[17] M. Sumetsky, “Optical microbubble resonator”, Opt. Lett. 35, pp. 898(2010). 